\title{
Difference Equations and Sharing Values Concerning Entire Functions and Their Difference
}

\author{
Zhiqiang Mao ${ }^{1}$ and Huifang Liu $^{2}$ \\ ${ }^{1}$ School of Mathematics and Computer, Jiangxi Science and Technology Normal University, Nanchang 330038, China \\ ${ }^{2}$ College of Mathematics and Information Science, Jiangxi Normal University, Nanchang 330022, China \\ Correspondence should be addressed to Huifang Liu; liuhuifang73@sina.com
}

Received 19 January 2014; Accepted 22 March 2014; Published 7 April 2014

Academic Editor: Zong-Xuan Chen

Copyright (c) 2014 Z. Mao and H. Liu. This is an open access article distributed under the Creative Commons Attribution License, which permits unrestricted use, distribution, and reproduction in any medium, provided the original work is properly cited.

The value distribution of solutions of certain difference equations is investigated. As its applications, we investigate the difference analogue of the Brück conjecture. We obtain some results on entire functions sharing a finite value with their difference operators. Examples are provided to show that our results are the best possible.

\section{Introduction and Main Results}

In this paper, the term meromorphic function will mean being meromorphic in the whole complex plane $\mathbb{C}$. It is assumed that the reader is familiar with the standard notations and the fundamental results of the Nevanlinna theory; see, for example, [1-3]. In addition, we use notations $\sigma(f), \lambda(f)$ to denote the order and the exponent of convergence of the sequence of zeros of a meromorphic function $f$, respectively. The notation $S(r, f)$ is defined to be any quantity satisfying $S(r, f)=o(T(r, f))$ as $r \rightarrow \infty$, possibly outside a set $E$ of $r$ of finite logarithmic measure.

Let $f$ and $g$ be two nonconstant meromorphic functions, and let $a \in \mathbb{C}$. We say that $f$ and $g$ share $a \mathrm{CM}$, provided that $f-a$ and $g-a$ have the same zeros with the same multiplicities. Similarly, we say that $f$ and $g$ share $a$ IM, provided that $f-a$ and $g-a$ have the same zeros ignoring multiplicities.

The famous results in the uniqueness theory of meromorphic functions are the $5 \mathrm{IM}$ and $4 \mathrm{CM}$ shared values theorems due to Nevanlinna [4]. It shows that if two nonconstant meromorphic functions $f$ and $g$ share five different values IM or four different values CM, then $f \equiv g$ or $f$ is a linear fractional transformation of $g$. Condition $4 \mathrm{CM}$ shared values have been improved to $2 \mathrm{CM}+2 \mathrm{IM}$ by Gundersen [5], while the case $1 \mathrm{CM}+3 \mathrm{IM}$ still remains an open problem. Specifically, Brück posed the following conjecture.
Conjecture 1 (see [6]). Let $f$ be a nonconstant entire function satisfying the hyperorder $\sigma_{2}(f)<\infty$, where $\sigma_{2}(f)$ is not a positive integer. If $f$ and $f^{\prime}$ share a finite value a $C M$, then $f-a \equiv c\left(f^{\prime}-a\right)$ for some nonzero constant $c$.

In [6], Brück proved that the conjecture is true provided that $a=0$ or $N\left(r, 1 / f^{\prime}\right)=S(r, f)$. He also gave counterexamples to show that the restriction on the growth of $f$ is necessary.

In recent years, as the research on the difference analogues of Nevanlinna theory is becoming active, lots of authors [7-11] started to consider the uniqueness of meromorphic functions sharing values with their shifts or their difference operators.

Heittokangas et al. proved the following result which is a shifted analogue of Brück's conjecture.

Theorem A (see [8]). Let $f$ be a meromorphic function of $\sigma(f)<2$ and $\eta$ a nonzero complex number. If $f(z)$ and $f(z+\eta)$ share a finite value $a$ and $\infty C M$, then

$$
\frac{f(z+\eta)-a}{f(z)-a}=\tau,
$$

for some constant $\tau$.

In [8], Heittokangas et al. gave the example $f(z)=e^{z^{2}}+1$ which shows that $\sigma(f)<2$ cannot be relaxed to $\sigma(f) \leq 2$. 
For a nonzero complex number $\eta$, we define difference operators as

$$
\begin{aligned}
& \Delta_{\eta} f(z)=f(z+\eta)-f(z), \\
& \Delta_{\eta}^{n} f(z)=\Delta_{\eta}^{n-1}\left(\Delta_{\eta} f(z)\right), \quad n \in \mathbb{N}, n \geq 2 .
\end{aligned}
$$

Regarding the difference analogue of Brück's conjecture, we mention the following results.

Theorem B (see [7]). Let $f$ be a finite order transcendental entire function which has a finite Borel exceptional value $a$, and let $\eta$ be a constant such that $f(z+\eta) \not \equiv f(z)$. If $f(z)$ and $\Delta_{\eta} f(z)$ share a $C M$, then

$$
a=0, \quad \frac{f(z+\eta)-f(z)}{f(z)}=c,
$$

for some nonzero constant c.

Theorem C (see [11]). Let $f$ be a nonperiodic transcendental entire function of finite order. If $f(z)$ and $\Delta_{\eta}^{n} f(z)$ share a nonzero finite value a $C M$, then $1 \leq \sigma(f) \leq \lambda(f-a)+1$; that is,

$$
f(z)=A(z) e^{Q(z)}+a,
$$

where $A(z)$ is an entire function with $\sigma(A)=\lambda(f-a)$ and $Q(z)$ is a polynomial with $\operatorname{deg} Q \leq \sigma(A)+1$.

Let $f$ be a nonperiodic transcendental entire function of finite order. Theorem $B$ shows that if a nonzero finite value $a$ is shared by $f(z)$ and $\Delta_{\eta} f(z)$, then $\sigma(f)=\lambda(f-a)$. It is obvious that the result in Theorem B is sharper than Theorem $\mathrm{C}$ for $n=1$. In this paper, we continue to investigate the difference analogue of Brück's conjecture and obtain the following result.

Theorem 2. Let $f$ be a finite order entire function, $n \geq 2$ an integer, and $\eta$ a constant such that $\Delta_{\eta}^{n} f(z) \not \equiv 0$. If $f(z)$ and $\Delta_{\eta}^{n} f(z)$ share a finite value a $(\neq 0) C M$, then $\lambda(f-a)=\sigma(f) \geq$ 1 ; that is,

$$
f(z)=A(z) e^{Q(z)}+a,
$$

where $A(z)$ is an entire function with $1 \leq \sigma(A)=\lambda(f-a)=$ $\sigma(f)$ and $Q(z)$ is a polynomial with $\operatorname{deg} Q \leq \sigma(A)$.

Remark 3. It is obvious that Theorem 2 is sharper than Theorem $\mathrm{C}$ and a supplement of Theorem $\mathrm{B}$ for $n \geq 2$.

The discussions in Theorems $\mathrm{C}$ and 2 are concerning the case that shared value $a \neq 0$. When $a=0$, we obtain the following result.

Theorem 4. Let $f$ be a finite order entire function, $n$ a positive integer, and $\eta$ a constant such that $\Delta_{\eta}^{n} f(z) \neq \equiv$. If $f(z)$ and $\Delta_{\eta}^{n} f(z)$ share $0 C M$, then $1 \leq \sigma(f) \leq \lambda(f)+1$; that is,

$$
f(z)=A(z) e^{Q(z)},
$$

where $A(z)$ is an entire function with $\sigma(A)=\lambda(f)$ and $Q(z)$ is a polynomial with $\operatorname{deg} Q \leq \sigma(A)+1$.
It is well known that if a finite order entire function $f(z)$ shares a CM with $\Delta_{\eta}^{n} f(z)$, then $f(z)$ satisfies the difference equation

$$
\Delta_{\eta}^{n} f(z)-a=e^{Q(z)}(f(z)-a),
$$

where $Q(z)$ is a polynomial. Hence in order to prove the above results, we consider the value distribution of entire solutions of the difference equation

$$
\begin{gathered}
a_{n}(z) f(z+n \eta)+\cdots+a_{1}(z) f(z+\eta) \\
+\left(a_{0}(z)-e^{Q(z)}\right) f(z)=B(z)
\end{gathered}
$$

and obtain the following result.

Theorem 5. Let $a_{0}, \ldots, a_{n-1}, a_{n}(\equiv 0), B(\not \equiv 0)$ be polynomials, and let $Q$ be a polynomial with degree $m(\geq 1)$. Then every entire solution $f$ of finite order of (8) satisfies $\sigma(f) \geq m$ and

(i) if $\sigma(f)>1$, then $\lambda(f)=\sigma(f)$;

(ii) if $\sigma(f)=1$, then $\lambda(f)=\sigma(f)$ or $f$ has only finitely many zeros.

\section{Lemmas}

Lemma 6 (see [12]). Let $T:(0,+\infty) \rightarrow(0,+\infty)$ be a nondecreasing continuous function, $s>0, \alpha<1$, and let $F \subset \mathbb{R}^{+}$be the set of all $r$ such that $T(r) \leq \alpha T(r+s)$. If the logarithmic measure of $F$ is infinite, then

$$
\varlimsup_{r \rightarrow \infty} \frac{\log T(r)}{\log r}=\infty .
$$

Lemma 7 (see [13]). Let $f$ be a nonconstant meromorphic function of finite order, $\eta \in \mathbb{C}, \delta<1$. Then

$$
m\left(r, \frac{f(z+\eta)}{f(z)}\right)=o\left(\frac{T(r+|\eta|, f)}{r^{\delta}}\right)
$$

for all $r$ outside a possible exceptional set $E$ with finite logarithmic measure $\int_{E}(d r / r)<\infty$.

Remark 8. By Lemmas 6 and 7, we know that, for a nonconstant meromorphic function $f$ of finite order,

$$
m\left(r, \frac{f(z+\eta)}{f(z)}\right)=S(r, f) .
$$

Lemma 9 (see [3]). Let $f_{j}(j=1, \ldots, n+1)$ and $g_{j}(j=$ $1, \ldots, n)$ be entire functions such that

(i) $\sum_{j=1}^{n} f_{j}(z) e^{g_{j}(z)} \equiv f_{n+1}(z)$,

(ii) the order of $f_{j}$ is less than the order of $e^{g_{k}}$ for $1 \leq j \leq$ $n+1,1 \leq k \leq n$; and furthermore, the order of $f_{j}$ is less than the order of $e^{g_{h}-g_{k}}$ for $n \geq 2$ and $1 \leq j \leq n+1,1 \leq$ $h<k \leq n$.

Then $f_{j}(z) \equiv 0(j=1, \ldots, n+1)$. 
Lemma 10 (see [14]). Let $f$ be a meromorphic function with finite order $\sigma(f)=\sigma<1, \eta \in \mathbb{C} \backslash\{0\}$. Then for any given $\varepsilon>0$ and integers $0 \leq j<k$, there exists a set $E \subset(1, \infty)$ of finite logarithmic measure, so that, for all $|z|=r \notin E \bigcup[0,1]$, we have

$$
\left|\frac{\Delta_{\eta}^{k} f(z)}{\Delta_{\eta}^{j} f(z)}\right| \leq|z|^{(k-j)(\sigma-1)+\varepsilon} .
$$

Lemma 11 (see [15]). Let $a_{0}(z), \ldots, a_{k}(z)$ be entire functions with finite order. If there exists an integer $l(0 \leq l \leq k)$ such that

$$
\sigma\left(a_{l}\right)>\max _{\substack{0 \leq j \leq k \\ j \neq l}}\left\{\sigma\left(a_{j}\right)\right\}
$$

holds, then every meromorphic solution $f\left(\begin{array}{ll}\# & 0)\end{array}\right)$ of the difference equation

$$
a_{k}(z) f(z+k)+\cdots+a_{1}(z) f(z+1)+a_{0}(z) f(z)=0
$$

satisfies $\sigma(f) \geq \sigma\left(a_{l}\right)+1$.

\section{Proofs of Results}

Proof of Theorem 5. Let $f$ be an entire solution of finite order of (8). By Remark 8 and (8), we get

$$
\begin{aligned}
T\left(r, e^{\mathrm{Q}}\right)= & T\left(r, e^{\mathrm{Q}}-a_{0}\right)+S\left(r, e^{\mathrm{Q}}\right) \\
\leq & \sum_{j=1}^{n} m\left(r, \frac{f(z+j \eta)}{f(z)}\right) \\
& +\sum_{j=0}^{n} m\left(r, a_{j}\right)+m\left(r, \frac{B(z)}{f(z)}\right)+S\left(r, e^{\mathrm{Q}}\right) \\
\leq & T(r, f)+S(r, f)+S\left(r, e^{\mathrm{Q}}\right) .
\end{aligned}
$$

By (15) we get $\sigma(f) \geq m$.

Case $1(\sigma(f)>1)$. Suppose that $\lambda(f)<\sigma(f)$, by the Weierstrass factorization; we get $f(z)=h_{1}(z) e^{h_{2}(z)}$, where $h_{1}(z)(\neq \equiv 0)$ is an entire function and $h_{2}(z)$ is a polynomial such that

$$
\sigma\left(h_{1}\right)=\lambda\left(h_{1}\right)=\lambda(f)<\sigma(f)=\operatorname{deg} h_{2}
$$

Substituting $f(z)=h_{1}(z) e^{h_{2}(z)}$ into (8), we get

$$
\begin{aligned}
& \sum_{j=1}^{n} a_{j}(z) h_{1}(z+j \eta) e^{h_{2}(z+j \eta)-h_{2}(z)} \\
& \quad+\left(a_{0}(z)-e^{Q(z)}\right) h_{1}(z)=B(z) e^{-h_{2}(z)} .
\end{aligned}
$$

If $\operatorname{deg} h_{2}>m$, then by (16) we know that the order of the right side of (17) is deg $h_{2}$, and the order of the left side of (17) is less than $\operatorname{deg} h_{2}$. This is a contradiction. Hence $\operatorname{deg} h_{2}=m>1$. Set

$$
\begin{aligned}
& Q(z)=b_{m} z^{m}+\cdots+b_{0}, \\
& h_{2}(z)=c_{m} z^{m}+\cdots+c_{0},
\end{aligned}
$$

where $b_{m}(\neq 0), \ldots, b_{0}, c_{m}(\neq 0), \ldots, c_{0}$ are complex numbers. By (17) we get

$$
\begin{aligned}
& \sum_{j=1}^{n} a_{j}(z) h_{1}(z+j \eta) e^{h_{2}(z+j \eta)-h_{2}(z)} \\
& \quad+a_{0}(z) h_{1}(z)=h_{1}(z) e^{Q(z)}+B(z) e^{-h_{2}(z)} .
\end{aligned}
$$

Next we discuss the following two subcases.

Subcase $1\left(b_{m}+c_{m} \neq 0\right)$. Then by Lemma 9, (16), and (19), we get $B(z) \equiv 0, h_{1}(z) \equiv 0$. This is impossible.

Subcase $2\left(b_{m}+c_{m}=0\right)$. Suppose that

$$
h_{1}(z) e^{\mathrm{Q}(z)-b_{m} z^{m}}+B(z) e^{-h_{2}(z)-b_{m} z^{m}} \equiv 0 .
$$

Then $h_{1}(z)=-B(z) e^{-h_{2}(z)-Q(z)}$. By $\sigma\left(h_{1}\right)=\lambda\left(h_{1}\right)$, we obtain that $e^{-h_{2}(z)-\mathrm{Q}(z)}$ is a nonzero constant. Hence $h_{1}(z)$ is a nonzero polynomial. By (19) we get

$$
\begin{aligned}
& \sum_{j=1}^{n} a_{j}(z) h_{1}(z+j \eta) e^{h_{2}(z+j \eta)-h_{2}(z)} \\
& =-a_{0}(z) h_{1}(z) .
\end{aligned}
$$

Since $\operatorname{deg}\left\{h_{2}(z+j \eta)-h_{2}(z+i \eta)\right\}=m-1>0$ for $i \neq j$, then by Lemma 9 and (21), we get

$$
a_{j}(z) h_{1}(z+j \eta) \equiv 0 \quad(j=0,1, \ldots, n) .
$$

This is impossible. Hence we have $h_{1}(z) e^{Q(z)-b_{m} z^{m}}+$ $B(z) e^{-h_{2}(z)-b_{m} z^{m}} \neq \equiv$. Then from the order consideration, we know that the order of the right side of (19) is $m$, and the order of the left side of (19) is less than $m$. This is a contradiction. Hence $\lambda(f)=\sigma(f)$.

Case $2(\sigma(f)=1)$. Then by $\sigma(f) \geq m$, we get $m=1$. Suppose that $f(z)$ has infinitely many zeros and $\lambda(f)<\sigma(f)$; by the Weierstrass factorization, we get

$$
f(z)=h_{3}(z) e^{\beta z},
$$

where $\beta(\neq 0)$ is a complex number and $h_{3}(z)(\neq \equiv 0)$ is an entire function such that

$$
\sigma\left(h_{3}\right)=\lambda\left(h_{3}\right)=\lambda(f)<1 .
$$

Let $Q(z)=b_{1} z+b_{0}$, where $b_{1}(\neq 0), b_{0}$ are complex numbers. Substituting $f(z)=h_{3}(z) e^{\beta z}$ into (8), we get

$$
\begin{gathered}
\sum_{j=1}^{n} a_{j}(z) h_{3}(z+j \eta) e^{\beta j \eta}+a_{0}(z) h_{3}(z) \\
=h_{3}(z) e^{b_{1} z+b_{0}}+B(z) e^{-\beta z} .
\end{gathered}
$$


Note that $h_{3}(z) e^{b_{0}}+B(z) \neq \equiv$; otherwise $f$ has only finitely many zeros. If $b_{1}+\beta=0$, then the order of the right side of (25) is 1 , but the order of the left side of (25) is less than 1 . This is absurd. If $b_{1}+\beta \neq 0$, then by Lemma $9,(24)$, and (25), we get $h_{3}(z) \equiv 0, B(z) \equiv 0$. This is impossible. Hence $\lambda(f)=\sigma(f)$. Theorem 5 is thus completely proved.

Proof of Theorem 2. Since $f(z)$ and $\Delta_{\eta}^{n} f(z)$ share $a \mathrm{CM}$ and $f$ is of finite order, then

$$
\frac{\Delta_{\eta}^{n} f(z)-a}{f(z)-a}=e^{\mathrm{Q}(z)},
$$

where $Q(z)$ is a polynomial with $\operatorname{deg} Q \leq \sigma(f)$. Now we will take two steps to complete the proof.

Step 1. We prove that $\lambda(f-a)=\sigma(f)$.

Let $F(z)=f(z)-a$; then

$$
\lambda(F)=\lambda(f-a), \quad \sigma(F)=\sigma(f) \geq \operatorname{deg} Q,
$$

and $\Delta_{\eta}^{n} f(z)=\Delta_{\eta}^{n} F(z)=\sum_{j=0}^{n}\left(\begin{array}{c}n \\ j\end{array}\right)(-1)^{n-j} F(z+j \eta)$. By this and (26), we get

$$
\sum_{j=1}^{n}\left(\begin{array}{c}
n \\
j
\end{array}\right)(-1)^{n-j} F(z+j \eta)+\left((-1)^{n}-e^{\mathrm{Q}(z)}\right) F(z)=a .
$$

Next we discuss the following three cases.

Case $1(\operatorname{deg} Q \geq 1$ and $\sigma(F)>\operatorname{deg} Q)$. Then $\sigma(F)>1$. By Theorem 5(i), (27), and (28), we get $\lambda(f-a)=\sigma(f)$.

Case $2(\operatorname{deg} Q \geq 1$ and $\sigma(F)=\operatorname{deg} Q)$. If $\sigma(F)=\operatorname{deg} Q>1$, then by Theorem 5(i), (27), and (28), we get $\lambda(f-a)=\sigma(f)$. If $\sigma(F)=\operatorname{deg} Q=1$, then by Theorem 5(ii) and (27), we obtain that $\lambda(f-a)=\sigma(f)$, or $F$ has only finitely many zeros.

If $F$ has only finitely many zeros, set

$$
F(z)=h_{1}(z) e^{b z},
$$

where $h_{1}(z)(\neq 0)$ is a polynomial and $b(\neq 0)$ is a complex number; then substituting (29) into (28), we get

$$
\begin{aligned}
& \sum_{j=1}^{n}\left(\begin{array}{c}
n \\
j
\end{array}\right)(-1)^{n-j} h_{1}(z+j \eta) e^{b j \eta}+(-1)^{n} h_{1}(z) \\
& =h_{1}(z) e^{Q(z)}+a e^{-b z} .
\end{aligned}
$$

By (30) and $\Delta_{\eta}^{n} F(z) \neq \equiv$, we know that the order of the left side of (30) is 0 and the order of the right side of (30) is 1 unless $h_{1}(z)=-a$ and $Q(z)=-b z$. In this case, take it into the left side of (30); we have $(-a)\left(e^{b \eta}-1\right)^{n}=0$. Since all $a, b$, and $\eta$ are not zero, it is impossible. Hence we get $\lambda(f-a)=\sigma(f)$.

Case 3. $Q$ is a complex constant. Then by (28) we get

$$
\sum_{j=1}^{n}\left(\begin{array}{c}
n \\
j
\end{array}\right)(-1)^{n-j} F(z+j \eta)+\left((-1)^{n}-c\right) F(z)=a,
$$

where $c\left(=e^{Q} \neq 0\right)$ is a complex number. Suppose that $\lambda(F)<$ $\sigma(F)$. Let $F(z)=h_{2}(z) e^{h_{3}(z)}$, where $h_{2}(z)(\not \equiv 0)$ is an entire function and $h_{3}(z)$ is a polynomial such that

$$
\sigma\left(h_{2}\right)=\lambda\left(h_{2}\right)=\lambda(F)<\sigma(F)=\operatorname{deg} h_{3} .
$$

Substituting $F(z)=h_{2}(z) e^{h_{3}(z)}$ into (31), we get

$$
\begin{aligned}
& \sum_{j=1}^{n}\left(\begin{array}{c}
n \\
j
\end{array}\right)(-1)^{n-j} h_{2}(z+j \eta) e^{h_{3}(z+j \eta)-h_{3}(z)} \\
& +\left((-1)^{n}-c\right) h_{2}(z)=a e^{-h_{3}(z)} .
\end{aligned}
$$

Since $\operatorname{deg}\left(h_{3}(z+j \eta)-h_{3}(z)\right)=\operatorname{deg} h_{3}(z)-1,(j=1, \ldots, n)$, by (32) we obtain that the order of the left side of (33) is less than $\operatorname{deg} h_{3}$ and the order of the right side of (33) is $\operatorname{deg} h_{3}$. This is absurd. Hence we get $\lambda(f-a)=\sigma(f)$.

Step 2. We prove that $\sigma(f) \geq 1$.

Suppose that $\sigma(f)<1$. Since $f(z)$ and $\Delta_{\eta}^{n} f(z)$ share $a$ $\mathrm{CM}$, then

$$
\frac{\Delta_{\eta}^{n} f(z)-a}{f(z)-a}=c,
$$

where $c$ is a nonzero constant. Let $F(z)=f(z)-a$; then by (34) we get

$$
\Delta_{\eta}^{n} F(z)=c F(z)+a .
$$

Differentiating (35), we get

$$
\left(\Delta_{\eta}^{n} F(z)\right)^{\prime}=c F^{\prime}(z)
$$

Note that $\left(\Delta_{\eta}^{n} F(z)\right)^{\prime}=\Delta_{\eta}^{n}\left(F^{\prime}(z)\right)$ and $\sigma\left(F^{\prime}\right)=\sigma(F)=\sigma(f)<$ 1 . So by Lemma 10 and (36), we get

$$
|c|=\left|\frac{\Delta_{\eta}^{n}\left(F^{\prime}(z)\right)}{F^{\prime}(z)}\right| \leq|z|^{n(\sigma(F)-1)+\varepsilon} \longrightarrow 0 .
$$

This is absurd. So $\sigma(f) \geq 1$. Theorem 2 is thus completely proved.

Proof of Theorem 4. Since $f(z)$ and $\Delta_{\eta}^{n} f(z)$ share $0 \mathrm{CM}$ and $f$ is of finite order, then

$$
\frac{\Delta_{\eta}^{n} f(z)}{f(z)}=e^{\mathrm{Q}(z)},
$$

where $Q(z)$ is a polynomial. By $\Delta_{\eta}^{n} f=\sum_{j=0}^{n}\left(\begin{array}{c}n \\ j\end{array}\right)(-1)^{n-j} f(z+$ $j \eta)$ and (38), we get

$$
\begin{aligned}
& f(z+n \eta) \\
& +\sum_{j=1}^{n-1}\left(\begin{array}{c}
n \\
j
\end{array}\right)(-1)^{n-j} f(z+j \eta) \\
& +\left((-1)^{n}-e^{Q(z)}\right) f(z)=0 .
\end{aligned}
$$


We discuss the following two cases.

Case 1. $Q$ is a polynomial with $\operatorname{deg} Q=m \geq 1$. Then by Lemma 11 and (39), we get $\sigma(f) \geq m+1$. Now we prove $\sigma(f) \leq \lambda(f)+1$. Suppose that $\sigma(f)>\lambda(f)+1$; then by the Weierstrass factorization, we get $f(z)=h_{1}(z) e^{h_{2}(z)}$, where $h_{1}(z)(\neq \equiv 0)$ is an entire function and $h_{2}(z)$ is a polynomial such that

$$
\begin{gathered}
\sigma\left(h_{1}\right)=\lambda\left(h_{1}\right)=\lambda(f), \\
\sigma(f)=\operatorname{deg} h_{2}>\sigma\left(h_{1}\right)+1 .
\end{gathered}
$$

Substituting $f(z)=h_{1}(z) e^{h_{2}(z)}$ into (39), we get

$$
\begin{aligned}
& \sum_{j=1}^{n}\left(\begin{array}{c}
n \\
j
\end{array}\right)(-1)^{n-j} h_{1}(z+j \eta) e^{h_{2}(z+j \eta)-h_{2}(z)} \\
& +(-1)^{n} h_{1}(z)=h_{1}(z) e^{Q(z)} .
\end{aligned}
$$

If $\sigma(f)>m+1$, then by (40), (41), and $\operatorname{deg}\left(h_{2}(z+j \eta)-\right.$ $\left.h_{2}(z)\right)=\operatorname{deg} h_{2}(z)-1,(j=1, \ldots, n)$, we obtain that the order of the left side of (41) is $\operatorname{deg} h_{2}-1$ and the order of the right side of (41) is less than $\operatorname{deg} h_{2}-1$. This is absurd.

If $\sigma(f)=m+1$, then by (41) we get

$$
\begin{gathered}
\sum_{j=1}^{n}\left(\begin{array}{c}
n \\
j
\end{array}\right)(-1)^{n-j} h_{1}(z+j \eta) e^{h_{2}(z+j \eta)-h_{2}(z)} \\
-h_{1}(z) e^{Q(z)}=(-1)^{n+1} h_{1}(z) .
\end{gathered}
$$

Set

$$
h_{2}(z)=d_{m+1} z^{m+1}+\cdots+d_{0},
$$

where $d_{m+1}(\neq 0), \ldots, d_{0}$ are complex numbers. Then

$$
\begin{array}{r}
h_{2}(z+j \eta)-h_{2}(z) \\
=d_{m+1}(m+1) j \eta z^{m}+\cdots+d_{1} j \eta, \\
\quad(j=1, \ldots, n) .
\end{array}
$$

Now we discuss the following two subcases.

Subcase $1 . \operatorname{deg}\left(Q(z)-\left(h_{2}(z+j \eta)-h_{2}(z)\right)\right)=m$ holds for every $j \in\{1, \ldots, n\}$. Then by $(40),(42), \operatorname{deg}\left(h_{2}(z+j \eta)-h_{2}(z+i \eta)\right)=$ $m,(j \neq i)$, and Lemma 9 , we get $h_{1}(z) \equiv 0$. This is absurd.

Subcase 2. There exist some $j_{0} \in\{1, \ldots, n\}$ such that $\operatorname{deg}\left(Q(z)-\left(h_{2}\left(z+j_{0} \eta\right)-h_{2}(z)\right)\right) \leq m-1$. Then by (44) we have $\operatorname{deg}\left(Q(z)-\left(h_{2}(z+j \eta)-h_{2}(z)\right)\right)=m$ for $j \neq j_{0}$. Merging the term $-h_{1}(z) e^{Q(z)}$ into $\left(\begin{array}{c}n \\ j_{0}\end{array}\right)(-1)^{n-j_{0}} h_{1}\left(z+j_{0} \eta\right) e^{h_{2}\left(z+j_{0} \eta\right)-h_{2}(z)}$, by (42) we get

$$
\begin{aligned}
& \sum_{\substack{j=1 \\
j \neq j_{0}}}^{n}\left(\begin{array}{c}
n \\
j
\end{array}\right)(-1)^{n-j} h_{1}(z+j \eta) e^{h_{2}(z+j \eta)-h_{2}(z)} \\
& \quad+A(z) e^{h_{2}\left(z+j_{0} \eta\right)-h_{2}(z)} \\
& \quad=(-1)^{n+1} h_{1}(z), \quad(n \geq 2)
\end{aligned}
$$

or

$$
A(z) e^{h_{2}\left(z+j_{0} \eta\right)-h_{2}(z)}=(-1)^{n+1} h_{1}(z), \quad(n=1),
$$

where $A(z)=\left(\begin{array}{c}n \\ j_{0}\end{array}\right)(-1)^{n-j_{0}} h_{1}\left(z+j_{0} \eta\right)-h_{1}(z)$ $e^{\mathrm{Q}(z)-\left(h_{2}\left(z+j_{0} \eta\right)-h_{2}(z)\right)}$ satisfying $\sigma(A)<m$. If $n \geq 2$, then by (40), (45), $\operatorname{deg}\left(h_{2}(z+j \eta)-h_{2}(z+i \eta)\right)=m,(j \neq i)$, and Lemma 9 , we get $h_{1}(z) \equiv 0$. This is absurd. If $n=1$, then by (46) and $h_{1}(z) \neq \equiv 0$, we get $A(z) \neq \equiv 0$. By this we know that the order of the left side of (46) is $m$ and the order of the right side of (46) is less than $m$. This is absurd. Hence we get $\sigma(f) \leq \lambda(f)+1$.

Case 2. $Q$ is a complex constant. Then by Lemma 10 and (38), we get $\sigma(f) \geq 1$. Now we prove $\sigma(f) \leq \lambda(f)+1$. Suppose that $\sigma(f)>\lambda(f)+1$. If $\sigma(f)>1$, then by the similar argument to that of case 1 , we get $h_{1}(z) \equiv 0$. This is absurd. If $\sigma(f)=1$, then by (40) we get $0 \leq \lambda(f)<\sigma(f)-1=0$. Since $\lambda(f)=0$, then $\sigma(f)=\lambda(f)+1$. Theorem 4 is thus completely proved.

\section{Some Examples}

The following examples show the existence of such entire functions which satisfy Theorems 2-5. Moreover, Example 2 shows that the result in Theorem 4 is the best possible.

Example 1. Let $\eta=1, n=2$, and $f(z)=(d+1)^{z}+\left(\left(d^{2}-\right.\right.$ $\left.1) / d^{2}\right) a$, where $a(\neq 0), d(\neq 0, \pm 1)$ are constants. Then $f(z)$ and $\Delta_{\eta}^{n} f(z)$ share $a \mathrm{CM}$ and $\sigma(f)=\lambda(f-a)=1$.

Example 2. Let $\eta=1, n=2$, and $f(z)=e^{z}$. Then $f(z)$ and $\Delta_{\eta}^{n} f(z)$ share $0 \mathrm{CM}$ and $\sigma(f)=1=\lambda(f)+1$.

Example 3. Let $\eta=1, n=2$, and $f(z)=H(z) e^{z}$, where $H(z)$ is an entire function with period 1 such that $\sigma(H)>1$ and $\sigma(H) \notin \mathbb{N}$. Then $f(z)$ and $\Delta_{\eta}^{n} f(z)$ share $0 \mathrm{CM}$ and $\lambda(f)=$ $\lambda(H)=\sigma(H)=\sigma(f)>1$. (Ozawa [16] proved that for any $\sigma \in[1, \infty)$ there exists a period entire function of order $\sigma$.)

Example 4. The entire function $f(z)=z e^{-z}$ satisfies the difference equation

$$
f(z+2 \eta)-4 f(z+\eta)+\left(4-e^{z}\right) f(z)=-z,
$$

where $\eta=-\log 2$. Here $\sigma(f)=1$ and $f$ has only finitely many zeros.

\section{Conflict of Interests}

The authors declare that there is no conflict of interests regarding the publication of this paper.

\section{Acknowledgments}

This work is supported by the National Natural Science Foundation of China (nos. 11201195, 11171119) and the Natural Science Foundation of Jiangxi, China (nos. 20122BAB201012, 20132BAB201008.). 


\section{References}

[1] W. K. Hayman, Meromorphic Functions, Clarendon Press, Oxford, UK, 1964.

[2] I. Laine, Nevanlinna Theory and Complex Differential Equations, Walter de Gruyter, Berlin, Germany, 1993.

[3] C.-C. Yang and H.-X. Yi, Uniqueness Theory of Meromorphic Functions, Kluwer Academic Publishers, New York, NY, USA, 2003.

[4] R. Nevanlinna, Le Theoreme de Picard-Borel et la Theorie des Fonctions Meromorphes, Gauthiers-Villars, Paris, France, 1929.

[5] G. G. Gundersen, "Meromorphic functions that share four values," Transactions of the American Mathematical Society, vol. 277, no. 2, pp. 545-567, 1983.

[6] R. Brück, "On entire functions which share one value CM with their first derivative," Results in Mathematics, vol. 30, no. 1-2, pp. 21-24, 1996.

[7] Z.-X. Chen and H.-X. Yi, "On sharing values of meromorphic functions and their differences," Results in Mathematics, vol. 63, no. 1-2, pp. 557-565, 2013.

[8] J. Heittokangas, R. Korhonen, I. Laine, J. Rieppo, and J. Zhang, "Value sharing results for shifts of meromorphic functions, and sufficient conditions for periodicity," Journal of Mathematical Analysis and Applications, vol. 355, no. 1, pp. 352-363, 2009.

[9] J. Heittokangas, R. Korhonen, I. Laine, and J. Rieppo, "Uniqueness of meromorphic functions sharing values with their shifts," Complex Variables and Elliptic Equations, vol. 56, pp. 81-92, 2011.

[10] K. Liu and L.-Z. Yang, "Value distribution of the difference operator," Archiv der Mathematik, vol. 92, no. 3, pp. 270-278, 2009.

[11] S. Li and Z. Gao, "A note on the Brück conjecture," Archiv der Mathematik, vol. 95, no. 3, pp. 257-268, 2010.

[12] R. G. Halburd and R. J. Korhonen, "Nevanlinna theory for the difference operator," Annales Academice Scientiarum Fennicœ. Mathematica, vol. 31, no. 2, pp. 463-478, 2006.

[13] R. G. Halburd and R. J. Korhonen, "Difference analogue of the lemma on the logarithmic derivative with applications to difference equations," Journal of Mathematical Analysis and Applications, vol. 314, no. 2, pp. 477-487, 2006.

[14] Y.-M. Chiang and S.-J. Feng, "On the growth of logarithmic differences, difference quotients and logarithmic derivatives of meromorphic functions," Transactions of the American Mathematical Society, vol. 361, no. 7, pp. 3767-3791, 2009.

[15] Y.-M. Chiang and S.-J. Feng, "On the Nevanlinna characteristic of $f(z+\eta)$ and difference equations in the complex plane," Ramanujan Journal, vol. 16, no. 1, pp. 105-129, 2008.

[16] M. Ozawa, "On the existence of prime periodic entire functions," Kōdai Mathematical Seminar Reports, vol. 29, no. 3, pp. 308-321, 1978. 


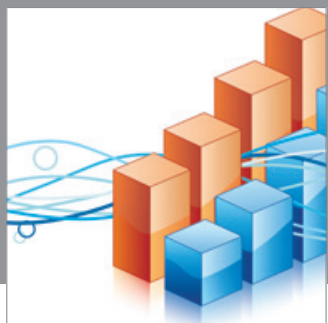

Advances in

Operations Research

mansans



The Scientific World Journal
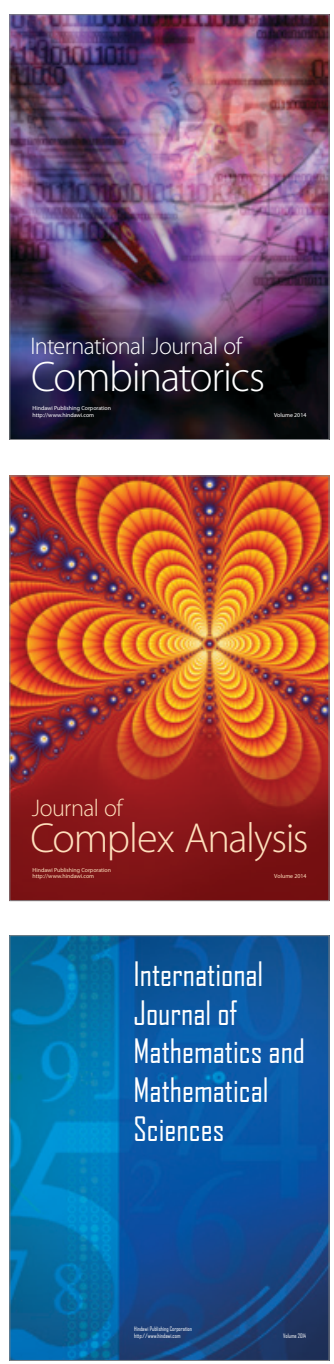
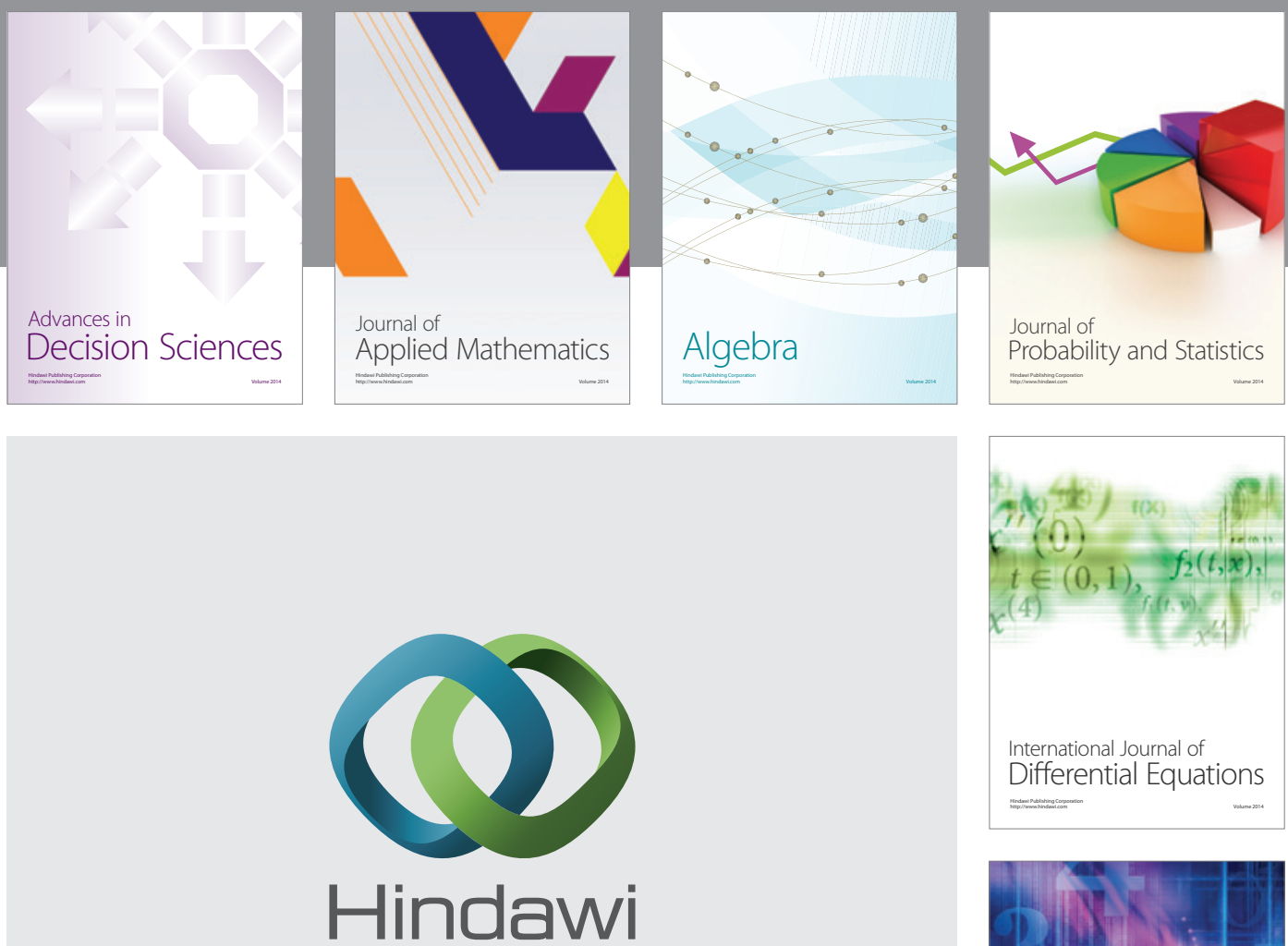

Submit your manuscripts at http://www.hindawi.com
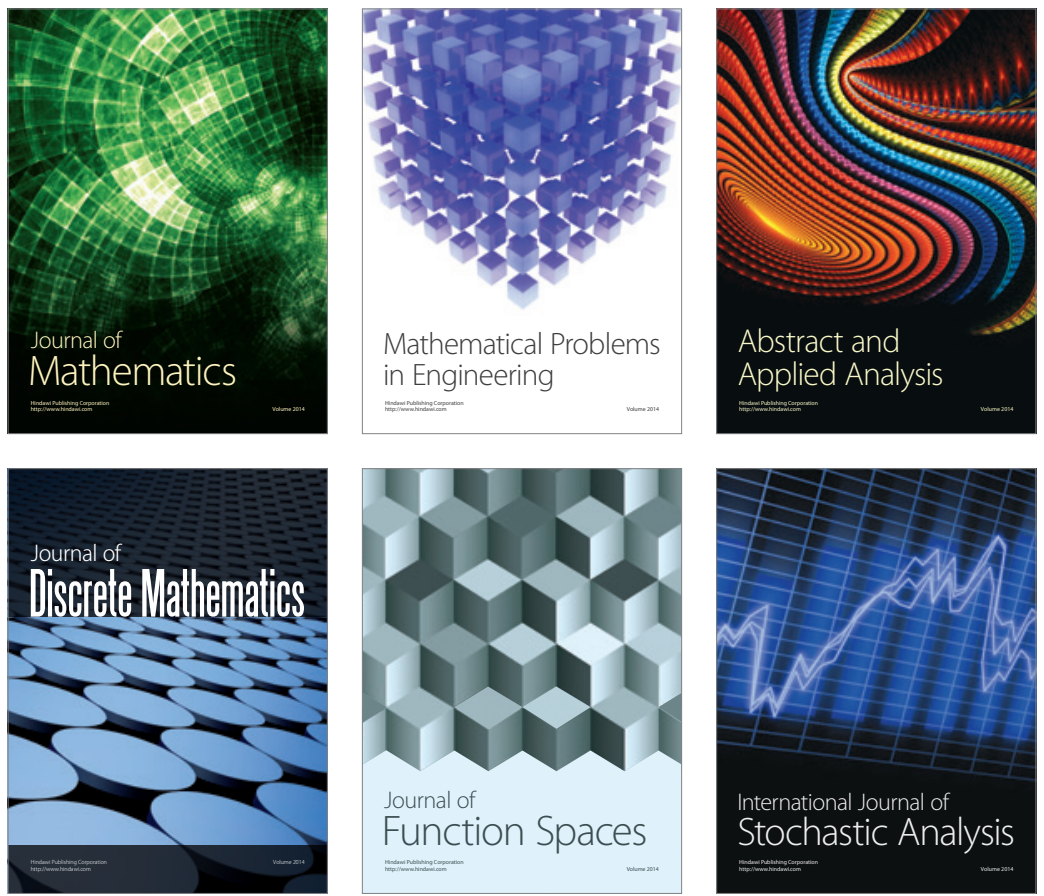

Journal of

Function Spaces

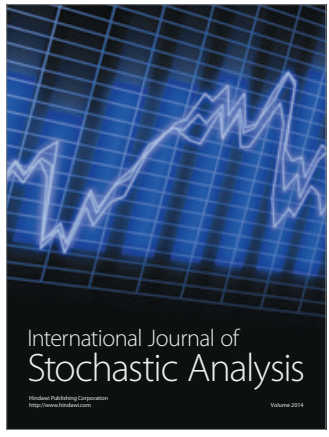

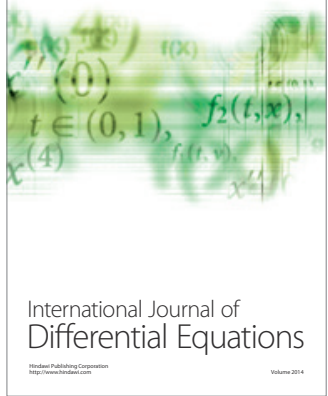
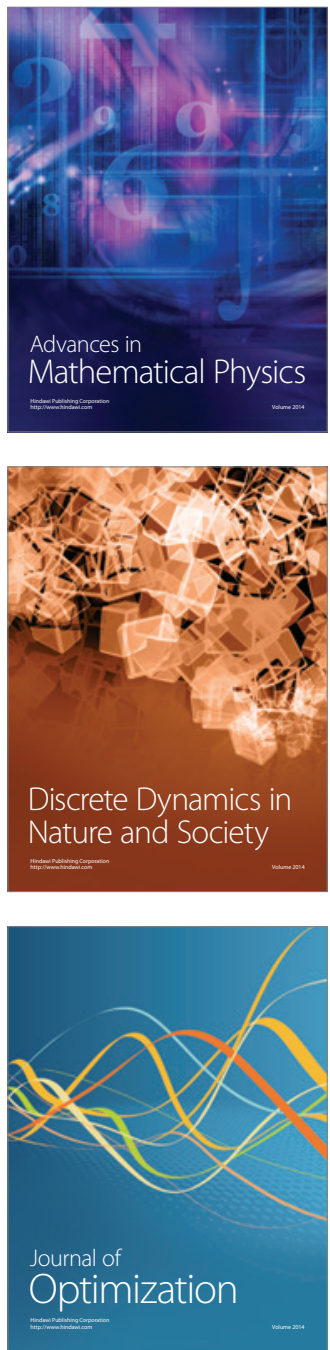